\title{
Les Stéréotypes : stéréotypes de pensée et stéréotypes de langue
}

\author{
Charlotte Schapira \\ Technion - IIT, Haïfa \\ gsrchar@tx.technion.ac.il
}

\section{Introduction}

Depuis trois décennies, un nombre impressionnant de travaux a été consacré, directement ou indirectement, à la stéréotypie linguistique. Directement, afin de cerner le phénomène, tenter de le définir et d'en explorer les origines, le développement, les caractéristiques et le fonctionnement ; indirectement, parce que la stéréotypie recoupe ou carrément recouvre d'autres phénomènes linguistiques ayant particulièrement suscité l'intérêt des chercheurs : la lexicalisation, le figement - et la phraséologie, à laquelle aboutissent ces processus.

Un lecteur assidu de cette vaste littérature ne peut manquer d'y observer des questions de plus en plus fréquentes concernant les rapports entre le stéréotype de pensée et le stéréotype de langue. Elles traduisent dans beaucoup de cas une certaine perplexité quant à la frontière entre les deux concepts et, occasionnellement, une presque totale confusion quant à leur signification.

L'idée qui se dégage, explicite ou implicite ${ }^{1}$, de bon nombre de textes consacrés à ces phénomènes linguistiques est que la phraséologie en général et les stéréotypes de langue en particulier véhiculent pour la plupart des stéréotypes de pensée : des idées rebattues, des opinions et des jugements non vérifiés. Elle impute donc, indirectement, la stéréotypie linguistique à une invasion de la langue par la doxa envisagée comme l'opinion commune - un fourre-tout contenant toutes les notions et les idées circulant dans la communauté locutrice de la langue. Cette conception de la doxa entraîne cependant des conséquences significatives quant à la manière de percevoir le fonctionnement même de la langue, parce qu'elle semble autoriser le raisonnement suivant : Les stéréotypes de langue véhiculent des stéréotypes de pensée. Or la langue est un outil cognitif primordial : elle forme et façonne, chez chaque individu, les représentations mentales du mondain. Donc, les stéréotypes de langue transmettent et impriment aux usagers les stéréotypes de pensée dont ils sont porteurs.

C'est la conclusion de ce raisonnement que je me propose de vérifier dans ce qui suit ; car, si la relation entre la stéréotypie de pensée et la doxa a été assez largement étudiée, notamment dans le domaine de la rhétorique et de l'argumentation, ses rapports avec la stéréotypie linguistique n'ont encore jamais inspiré de recherches systématiques. Est-ce que tous les stéréotypes de langue reposent sur des stéréotypes de pensée ? Et, si tel est le cas, en quoi et jusqu'à quel point constituent-ils des stéréotypes de pensée ? Est-il vrai, par ailleurs, que les expressions figées lexicalisées fixent les stéréotypes de pensée dans l'esprit des usagers de la langue, renforçant ainsi leur pérennité dans la doxa? 
Voilà quelques-unes des questions auxquelles je tenterai de réfléchir, avec l'espoir, sinon d'y répondre, d'y apporter au moins quelques éclaircissements. Il semble utile, pour ce faire, de reprendre la question sur d'autres bases; d'introduire dans la discussion des facteurs certes pas nouveaux mais auxquels on donnerait plus de poids que dans les analyses précédentes.

Je commencerai donc par la définition, bien connue du stéréotype, pour m'interroger ensuite, rapidement, sur la stéréotypie de pensée et ses relations avec la doxa, puis sur le stéréotype de langue, sur les relations entre ces deux stéréotypies, avant d'aborder, enfin, la question centrale de l'adhésion de l'usager aux formules figées lexicalisées.

\section{Le stéréotype}

Le stéréotype est ainsi défini par le $\operatorname{TLF}^{2}$ (A 1):

«Cliché métallique en relief obtenu, à partir d'une composition en relief originale (caractères typographiques, gravure, photogravure, etc.), au moyen de flans qui prennent l'empreinte de la composition et dans lesquels on coule un alliage à base de plomb [...].»

Il devient ainsi synonyme de cliché, adopté au XIXe siècle pour le négatif de la photographie dont on pouvait tirer par la suite des images en série. Le TLF mentionne ensuite une autre acception (B 1), présentée comme un terme figuré, appartenant à la psychologie et à la sociologie :

«Idée, opinion toute faite, acceptée sans réflexion et répétée sans avoir été soumise à un examen critique (par un groupe ou toute une communauté linguistique) $[\ldots]$ et qui détermine, à un degré plus ou moins élevé, ses manières de penser, de sentir et d'agir.»

Puis (B 3), le sens du mot en linguistique et stylistique :

« Association stable d'éléments, groupe de mots formant une unité devenue indécomposable, réemployée après avoir perdu toute expressivité [...]. »

Il ne semble pas nécessaire de s'attarder sur le passage du littéral au figuré de ce terme. Soulignons cependant que ce passage inclut un glissement du neutre au péjoratif; et il n'est pas peu étonnant que ce moyen technique qui a permis le développement de la typographie et qui représente historiquement l'un des plus grands progrès de l'humanité, soit devenu au figuré un mot péjoratif. La chute évaluative provient sans doute du fait que, sorti de son contexte technique, le terme stéréotype met en opposition la banalisation obtenue par la production en série et le caractère original du prototype, qui est unique.

Une autre observation d'égale importance s'impose : les trois sens dominants indiqués par le TLF comme appartenant à des domaines d'activité différents (imprimerie, psychologie/sociologie, linguistique/stylistique) représentent en réalité, grosso modo, les définitions du stéréotype dans son sens littéral, du stéréotype de pensée et du stéréotype de langue. C'est en effet avec le sens donné en B 2 
que le vocable figure dans la collocation stéréotype de pensée. Celui-ci serait-il donc, ainsi que l'indique le dictionnaire et tant d'autres ouvrages qui l'étudient ou y font seulement référence, une idée toute faite, le «prêt-à-penser »? En réalité, le stéréotype de pensée est l'un des composants de la doxa - l'opinion commune. Il est donc indispensable, avant d'aller plus loin, de consacrer quelques mots à la doxa et à la place qu'il y occupe.

\section{De quoi est faite la doxa?}

La doxa est définie dans le $\mathrm{DAD}^{3}$ comme «l'opinion, la réputation, ce que l'on dit des choses et des gens ». En effet, doxa ${ }^{4}$ est un terme générique pour un ensemble d'idées et d'opinions en vigueur à un moment historique précis dans une communauté régionale, nationale ou transnationale, voire, pour certains de ses éléments, dans le monde entier. La doxa - les divers chercheurs qui l'ont étudiée le reconnaissent - a été et reste une notion floue ${ }^{5}$. Le terme, d'origine grecque, remontant à Platon et repris par Aristote, désigne un ensemble d'opinions, de jugements et de croyances, qui sont, toutes, des représentations collectives de la réalité ; dans la conception aristotélicienne doxa s'oppose à épistêmê, le corps organisé des connaissances théoriques : la science. C'est précisément cette opposition qui est à l'origine de la mauvaise réputation de la doxa. Réseau de connaissances élémentaires et triviales d'une part et, d'autre part, amalgame d'opinions et de croyances non vérifiées et souvent non vérifiables, elle serait même nécessairement porteuse d'une idéologie, et de ce fait susceptible d'être asservie à un courant de pensée dominant.

La doxa constitue cependant le produit obligé et indispensable de la pensée humaine ; elle est le résultat de la pensée individuelle se fondant dans la pensée collective, source des idées et des représentations du monde communes à tous les membres de la communauté. Les analystes du discours soulignent le rôle fondamental de ces savoirs « constitutifs de l'environnement cognitif et condition de l'intercompréhension sur la base d'un principe très général de pertinence »(Fournier 2010 :49). Domaine vaste et hétérogène, la doxa a été étudiée par des chercheurs de tous bords. Je me limiterai à en donner ici un aperçu rapide (forcément schématique et certainement peu orthodoxe) pour les besoins de mon propos. Mon intérêt se centre en effet sur une couche spécifique de la doxa : la première, la plus basse (dans le sens spatial du mot) et aussi la plus fondamentale. C'est cette strate qui sous-tend le lexique ; car, si la doxa s'exprime principalement par la langue ${ }^{6}$, seule cette base fondamentale est inscrite dans la langue.

Grosso modo, l'on peut y distinguer deux catégories génériques :

- le savoir partagé : un savoir primaire et fondamental, qui ne s'apprend pas à l'école et s'acquiert très tôt dans la vie ;

- l'ensemble des opinions sur tout ce qui est humain.

\subsection{Le savoir partagé}

Ainsi que le remarque Sarfati (2000:40), « à partir d'Aristote, l'histoire de la philosophie nous engage à distinguer entre deux notions (doxales). La première renvoie [...] à une rationalité commune, [...] faculté organisatrice des données de la perception, alors que la seconde [...] réfère à l'opinion commune. » 
Cette première catégorie doxale, celle du savoir partagé, que certains appellent aussi «savoir commun » (Charaudeau, Fournier) ou « science populaire » (Anscombre), est le fondement sur lequel se construit et s'échafaude toute la connaissance individuelle acquise par un être humain au cours de sa vie. Elle correspond à ce que Paveau (2003) appelle « ce qui va sans dire », à ce que Sarfati (2002b:58) désigne comme "l'informulé que suppose toute formulation». Il s'agit des connaissances que l'on ne considère même pas comme telles, qui ont leur origine dans l'observation, l'expérience personnelle, l'acquisition des premiers principes existentiels, la tradition transmise verbalement et non verbalement; c'est la strate la plus élémentaire de la doxa, une strate pré-encyclopédique, qui précède celle de la compétence encyclopédique (Kerbrat-Orrecchioni, 1980), elle aussi doxale mais très variable d'un sujet à l'autre. Ce réservoir de connaissances et d'idées - «ce qui va sans dire »- est, en effet, ce dont on ne parle jamais : à quel propos mentionner que l'été il fait chaud et l'hiver il fait froid, que la glace est froide et le feu brûle, que les animaux sauvages sont dangereux, que les chats ne s'entendent pas avec les chiens, que le jour commence avec le lever du soleil et que la nuit il fait noir, etc. ? Et pourtant, c'est sur cette connaissance primaire (dans tous les sens du mot) du monde que reposent implicitement ou explicitement le folklore et tous les autres genres de création populaire, y compris le proverbe, qui nous intéressera ici. A ces acquis fondamentaux s'ajoutent aussi, par l'éducation et la lecture, les connaissances encyclopédiques que tout individu gagne au cours de son existence. Cette première catégorie de la doxa concerne le mondain et ses objets et aussi les humains mais seulement en tant qu'objets du monde: leurs caractéristiques « objectives »- couleur de la peau, morphologie, mais aussi leur habitat, leur mode de vie, leurs traditions, etc. Les données de ce savoir partagé ne sont pas mises en question et ne sont pas falsifiables, parce que les contre-exemples sont perçus comme des exceptions et traités comme tels. Un hiver doux ne fera changer à personne l'idée que l'hiver il fait froid, etc. Un chien affectueux ne nous poussera pas à ouvrir sans crainte une porte derrière laquelle un chien aboie, etc.

\subsection{L'opinion commune}

La deuxième catégorie doxale a pour objet exclusif les humains sous leurs aspects psychologiques et sociaux : leur vie, leur appartenance sociale, les relations entre eux, leurs liens avec la nature et le monde. Il ne s'agit plus d'un savoir objectif ou du moins que l'on croit objectif; celuici est le domaine des représentations collectives, source des opinions individuelles, des préjugés, des superstitions.

«Tout individu, en effet, dispose de schèmes d'interprétation de la réalité quotidienne qui lui permettent d'évoluer dans des situations courantes - écrit Fournier (2010:50). - Une partie importante de ces schèmes sont des éléments sémiotiques préfabriqués, emblématisés par la communauté qui les produits et s'en nourrit. Ces schèmes interprétatifs sont précisément les stéréotypes ». 
L'interaction verbale est donc sous-tendue par des préconstruits culturels, auxquels s'ajoutent, dans le schéma de la communication proposé par J.-M Adam $(1999)^{7}$, les préconstruits linguistiques. Ce sont ces idées reçues, ces préjugés, ces superstitions, qui engendrent le plus souvent la méfiance à l'égard de l'altérité, quelle qu'elle soit: physique, sociale, ethnique, religieuse, etc. A partir du savoir objectif par exemple: les Africains sont noirs - s'est développée historiquement l'idée que les Noirs sont inférieurs aux Blancs; dans certaines communautés les roux, les albinos, les handicapés passaient pour des êtres méchants, malveillants ou diaboliques.

Une autre source majeure de préjugés - la plus importante peut-être - réside dans la généralisation abusive.

« [...] les jugements stéréotypés consistent à ramener tous les membres d'un groupe à des caractéristiques générales, ou encore à les penser ou à les percevoir sous la catégorie de l'espèce $»^{8}$ - lit-on dans la Encyclopedia Universalis.

C'est dans cette catégorie que naissent et se propagent les stéréotypes de pensée dans le sens que leur donnent Amossy et Herschberg-Pierrot (1997): « le prêt-à-penser ». Le terme «stéréotype » est justifié dans ce contexte : une opinion, par définition individuelle, a fait tache d'huile, s'est généralisée et, par là même, s'est banalisée au cours du temps, au point d'être perçue comme une vérité indiscutable.

«Un stéréotype a donc la fonction [...] d'affirmer ou plutôt de confirmer des propositions paraissant évidentes aux yeux de la communauté. Ce maniement relève alors de la norme d'usage et ne nécessite pas forcément une explication $[. .] ;$.1 'explication peut rester allusive puisqu 'elle est inscrite dans l'usage collectif.' $\gg$ (Fournier 2010 :54)

Si le savoir partagé est « ce qui va sans dire », l'opinion commune est « ce qui va de soi ».

\section{Le stéréotype de pensée}

Notre conception du monde repose sur des représentations schématiques, plus ou moins complexes, selon les individus, et figées en stéréotypes. Toute modification, à plus forte raison l'effacement de la doxa d'une croyance, demande une longue période de temps, puisque la doxa survit le plus souvent aux instances qui l'ont engendrée; mais la prise de position individuelle face à une idée considérée comme erronée y contribue beaucoup. Un exemple historique illustre est la révolution doxale qui a suivi les découvertes de Copernic et de Galilée dans le domaine de l'astrophysique. Les deux composants doxaux fondamentaux - le savoir partagé et l'opinion commune - se retrouvent aussi à des niveaux supérieurs de la doxa, là où l'opinion commune, plus fine et plus insidieuse, s'insinue dans l'argumentation; où elle sous-tend le débat; où elle est susceptible de servir une idéologie. Ils se retrouvent par ailleurs aussi dans des doxas spécifiques : la doxa du social, de la politique ou même dans 
des doxas scientifiques particulières, comme la doxa juridique ou médicale. Les linguistes, notamment dans l'analyse du discours, préconisent, de ce fait, l'étude des phénomènes doxaux dans des tranches plus grandes de textes, voire dans des ouvrages entiers, ou la pensée doxale ne se livre que graduellement, se camouflant même, parfois, sous des effets de langue; c'est que le stéréotype de pensée n'a pas d'expression linguistique fixe. Il incombe donc à l'analyste du discours de le démasquer, de le formuler explicitement et de le démonter en tant qu'argument.

Le stéréotype de pensée, l'opinion commune qui ne fait pas l'objet d'un examen critique est donc, à juste titre, perçu comme une notion péjorative.

Plus récemment, cependant, dans le cadre des études sur la communication en général et sur la communication verbale en particulier, la doxa et le stéréotype sont traités comme des concepts neutres, produits naturels, obligés et, qui plus est, nécessaires à l'intégration de l'individu dans la communauté linguistique qui les a produits. De nombreux travaux, notamment dans le domaine de la sociologie et de la sociolinguistique mettent en évidence le rôle positif de la doxa en tant que territoire commun favorisant la discussion, le débat, l'argumentation.

\section{Le stéréotype de langue}

Disons-le d'emblée : le stéréotype de langue ne constitue pas, comme certains l'entendent, la contrepartie du stéréotype de pensée : le terme est purement technique et totalement neutre, puisque "stéréotype » y figure avec sa signification originelle : un modèle initialement unique qui, en se multipliant, a perdu son originalité. Cependant, avant de discuter les rapports entre ce genre de stéréotype et le stéréotype de pensée, il convient de rappeler rapidement la définition du phénomène et ses manifestations.

Le stéréotype de pensée, nous l'avons dit, n'a pas d'expression linguistique fixe; il peut s'exprimer dans des segments de phrase, dans des phrases entières, des séquences plus larges de texte ou même dans des ouvrages complets. Remâcher «le déjà dit »- comme on a l'habitude d'appeler le stéréotype de pensée signifie répéter son contenu mais non nécessairement sous la même forme. Le stéréotype de pensée est susceptible d'être repris par différents locuteurs et même par un locuteur unique, de manières diverses et stylistiquement variées.

Les stéréotypes de langue présentent, au contraire, une forme fixe. Ils constituent, en français, une souscatégorie des expressions figées10, puisqu'ils consistent en des séquences de discours polylexicales, syntagmatiques ou phrastiques, qui, ayant d'abord été des combinaisons individuelles libres, se sont ensuite fixées dans l'usage. Leur originalité par rapport aux autres séquences figées réside dans le fait qu'elles formulent de manière expressive des concepts pour lesquels la langue offre la possibilité d'un mot ou d'une d'expression neutre, stylistiquement non marquée11. Une autre clarification à apporter d'emblée est que le stéréotype de langue n'est pas toujours porteur d'un stéréotype de pensée. Un très grand nombre de ces expressions (battre la campagne, prendre la clé des champs, avoir une araignée dans le plafond, avaler des couleuvres, prendre ses jambes à son cou, ne pas savoir à quel saint se vouer, mettre la charrue avant les bœufs, etc. - pour n'en citer que quelques-unes, au hasard) sont des tropes à valeur purement stylistique. En effet, paradoxalement, tout stéréotype de langue a d'abord été une trouvaille stylistique, une image qui initialement s'est propagée en discours précisément grâce à son expressivité et qui, érodée par l'usage, a fini par perdre son originalité ${ }^{12}$. Lexicalisée au cours du temps par l'emploi répété, la figure 
stéréotypée s'est intégrée à la langue, devenant ainsi un item lexical à part entière.

Le but de cet exposé n'étant pas la taxinomie des stéréotypes, il ne semble pas nécessaire de présenter en détail les différentes classes de formules ${ }^{13} \mathrm{ni}$ de reprendre la discussion sur leurs frontières souvent incertaines. Laissant de côté la division désormais classique basée sur le critère syntaxique - séquence syntagmatique / énoncé phrastique - mon intérêt se porte ici sur deux classes distinctes de formules, dont l'une s'articule grosso modo sur le proverbe (proverbes et dictons), l'autre comprenant les clichés, les lieux communs et les stéréotypes conversationnels. Cette distinction s'impose d'elle-même, puisque, comme on le verra plus loin, chacune de ces deux catégories se comporte différemment et véhicule des éléments doxaux caractéristiques. En effet, alors que les proverbes se nourrissent des deux composantes doxales mentionnées plus haut, les autres stéréotypes, indépendamment de leur structure, sont les véhicules privilégiés des idées reçues, des opinions et des jugements acceptés sans réflexion, par la seule inertie de la tradition.

\section{Proverbe et stéréotype de pensée}

Afin de vérifier, d'une part, comment et dans quelle mesure les proverbes constituent des réceptacles de stéréotypes de pensée et, d'autre part, afin de chercher à savoir s'ils façonnent nos représentations du monde, nous devrons, au préalable, examiner de plus près leur relation avec la doxa. Il faudra ensuite tenter de comprendre s'il est vrai que l'emploi des proverbes en discours prouve automatiquement l'adhésion à l'idée qui y est exprimée. Pour ce faire, il semble nécessaire de présenter d'abord, rapidement, le fonctionnement sémantique du proverbe.

\subsection{Le fonctionnement sémantique du proverbe}

Après un débat qui a duré presque deux décennies et auquel ont participé plus ou moins activement tous les parémiologues de langue française, il semblerait qu'un consensus ait été atteint autour de la représentation du sémantisme proverbial proposée par Tamba (2011). Cette représentation, remarquable par sa simplicité, postule que «[...] le sens proverbial repose sur le couplage d'un sens phrastique compositionnel et d'un sens formulaire conventionnel, lexicalisé » (Tamba 2011:114), reliés entre eux par une relation de transparence métaphorique. En d'autres termes, comme le précise Tamba (2011:119), «le sens proverbial résulte de la conjonction de deux sens qui, pris séparément, sont autonomes et complets ».

Le premier sens auquel se réfère Tamba correspond au sens littéral, compositionnel, de la phrase proverbe ; le second, généralement métaphorique, s'applique aux humains. Parfois, les deux sens peuvent coïncider ; c'est le cas des proverbes dits « littéraux »:

Mieux vaut faire envie que pitié.

Tel père tel fils. 
Cependant - on le sait - la plupart des proverbes prennent leur signification formulaire au moyen d'une translation de type métaphorique aux humains. Ainsi, dans

Petit à petit l'oiseau fait son nid (exemple de Tamba 2011:114)

l'image de l'oiseau qui construit son nid avec effort, revenant inlassablement à la charge, conduit au sens formulaire conventionnel : «avec de la persévérance on arrive à ses fins ».

\subsection{Savoir partagé et opinion commune dans les proverbes}

Comme je l'ai déjà dit, certains proverbes s'appuient sur un exemplum littéral tiré du savoir commun. La soi-disant « science populaire » nourrit en effet un grand nombre de formules, et en premier lieu les proverbes dits «météorologiques» exprimant des prévisions fondées sur l'expérience:

A la Chandeleur l'hiver se passe ou prend vigueur.

Petite pluie abat grand vent.

Noël au balcon, Pâques aux tisons.

ou les proverbes qui parlent des travaux, agricoles et autres, que leur sens formulaire soit littéral:

Le semer et la moisson ont leur temps et leur saison.

Labour d'été vaut fumier.

ou métaphorique :

Il faut battre le fer tant qu'il est chaud.

C'est en forgeant qu'on devient forgeron.

C'est toujours le savoir populaire qui se trouve à l'origine du nombre considérable de proverbes tautologiques exprimant l'évidence poussée parfois jusqu'à l'absurde.

Il n'y a pas de rose sans épines.

On ne fait pas d'omelette sans casser des œufs.

Il faut pousser la porte pour l'ouvrir.

Il faut semer pour

moissonner. Qui peut le

plus peut le moins.

Cependant, la plupart des proverbes ou des énoncés apparentés aux proverbes se nourrissent, au contraire, d'opinions communes passant pour des vérités tirées de l'expérience :

Qui se ressemble se rassemble.

Morte la fille mort le gendre.

Qui veut noyer son chien l'accuse de la rage.

Certains sont carrément des superstitions érigées en règles :

Mariage pluvieux, mariage heureux. 
Or, en examinant le type de message transmis par la phrase, on découvre que le comportement des proverbes fondés sur des opinions communes diffère à bien de points de vue de celui des proverbes dont l'origine est la science populaire. Cette différence qui, à ma connaissance, n'a encore jamais été signalée, sera soulignée dans les sections suivantes.

\subsection{Un mécanisme de modération doxale}

En tant que voix de la doxa, les proverbes - cela a été remarqué plus d'une fois - adoptent, du point de vue linguistique, la forme de la loi, se donnant ainsi pour des vérités absolues, indépendamment du degré de vérité réel de ce qu'ils assertent. Cependant, cette autorité formelle apparemment sans appel n'est qu'un trait illusoire : la doxa est en réalité un système vivant, doté d'un mécanisme régulateur interne, modérant le caractère absolu et définitif de ses assertions. Il y a d'abord, comme on sait, des proverbes antonymiques :

Tel père tel fils. / A père avare fils prodigue.

L'argent ne fait pas le bonheur. / Abondance de biens ne nuit pas.

Il existe aussi un nombre non négligeable de proverbes à la forme négative, polémique, dans lesquels l'idée doxale lexicalisée combat une autre idée doxale, présupposée dans la phrase, et également bien ancrée, sinon davantage, dans l'esprit des usagers de la langue : ainsi, le sens formulaire de L'habit ne fait pas le moine ( $\mathrm{Il}$ ne faut pas se fier aux apparences en jugeant les hommes ») s'oppose, par exemple, à un autre stéréotype de pensée :« On juge les gens d'après les signes apparents de leur appartenance sociale ».

Cette opinion commune s'est d'ailleurs proverbialisée en d'autres langues; en roumain, par exemple ( Haina îl face pe om» = L'habit fait l'homme) et en allemand («Kleider machen Leute »).

Une hirondelle ne fait pas le printemps s'oppose au symbole du printemps qu'est le retour des hirondelles.

Nul n'est prophète dans son pays part de l'idée que chaque pays a ses prophètes.

Cependant, on remarquera que seuls les proverbes dont le sens littéral reflète une opinion commune acceptent la transformation affirmatif / négatif. La raison réside, précisément, dans le fait que l'inverse est aussi une idée doxale qui, dans certaines conditions, aurait pu, elle aussi, se stéréotyper.

En effet, dans la plupart des cas, la version positive des proverbes à la forme négative pourrait fonctionner elle aussi comme un proverbe :

(C'est) L'habit (qui) fait le moine.

Une hirondelle annonce le printemps. / Une hirondelle est déjà le printemps. / Il suffit d'une hirondelle pour savoir qu'il est printemps.

Chaque pays a ses prophètes.

Les proverbes dont le sens littéral reflète le savoir populaire, eux, n'acceptent pas cette transformation:

*On peut faire des omelettes sans casser des œufs.

*Il ne faut pas semer pour moissonner. 


\subsection{Du stéréotype doxal au stéréotype linguistique et le retour à la doxa}

Puisque le vrai message du proverbe est le sens formulaire, c'est que l'idée doxale qu'il véhicule préexiste à la phrase porteuse du sens littéral. En d'autres termes, la doxa, trésor chargé des richesses formant " la sagesse de la nation », cherche à pérenniser dans la langue certains de ses préceptes.

Création populaire s'adressant à tous les locuteurs de la langue indépendamment de leur capacité intellectuelle et/ou de leur éducation, le proverbe puise ses images exemplaires dans la strate doxale la plus fondamentale : soit dans le réservoir du savoir commun, soit parmi les stéréotypes les plus simples et les plus motivés dans l'usage. Ce sont eux qui fournissent la proposition qui constitue le proverbe même et son sens littéral.

Un exemple illustrera le mouvement circulaire du sens du proverbe à partir de sa source doxale jusqu'au stéréotype de pensée réintégré à la doxa.

Tout proverbe a pour source une opinion. Considérons, par exemple, l'opinion commune que l'abus dans n'importe quel domaine risque d'entraîner des conséquences négatives. Pour l'illustrer, l'usage a imposé au cours du temps un fait puisé dans le savoir populaire, un de ces faits qui «vont sans dire $»$ : toute cruche, quelle que soit la période de temps qu'elle a servi, finit par se casser :

Tant va la cruche à l'eau qu'à la fin elle se casse.

Cette phrase se lexicalise et, en cristallisant son sens formulaire, fixe l'opinion initiale en la précisant : à force de s'exposer souvent à quelque danger ou tentation on risque d'y succomber ou d'en devenir la victime.

Dans d'autres cas cependant, l'opinion commune initiale (1 dans le schéma ci-dessous) a été illustrée par une autre opinion commune (2). Ainsi, l'idée doxale selon laquelle nous sommes nous-mêmes les artisans de notre destin a pris pour image exemplaire une autre opinion stéréotypée, plus concrète (que nous voyons d'ailleurs encore abondamment exploitée dans la publicité), à savoir que le choix du lit conditionne la qualité du repos:

Comme on fait son lit on se couche,

dont le sens formulaire, désormais inscrit dans la langue est : « on a du bien ou du mal suivant qu'on a eu prévoyance et précaution » (Littré)15. 


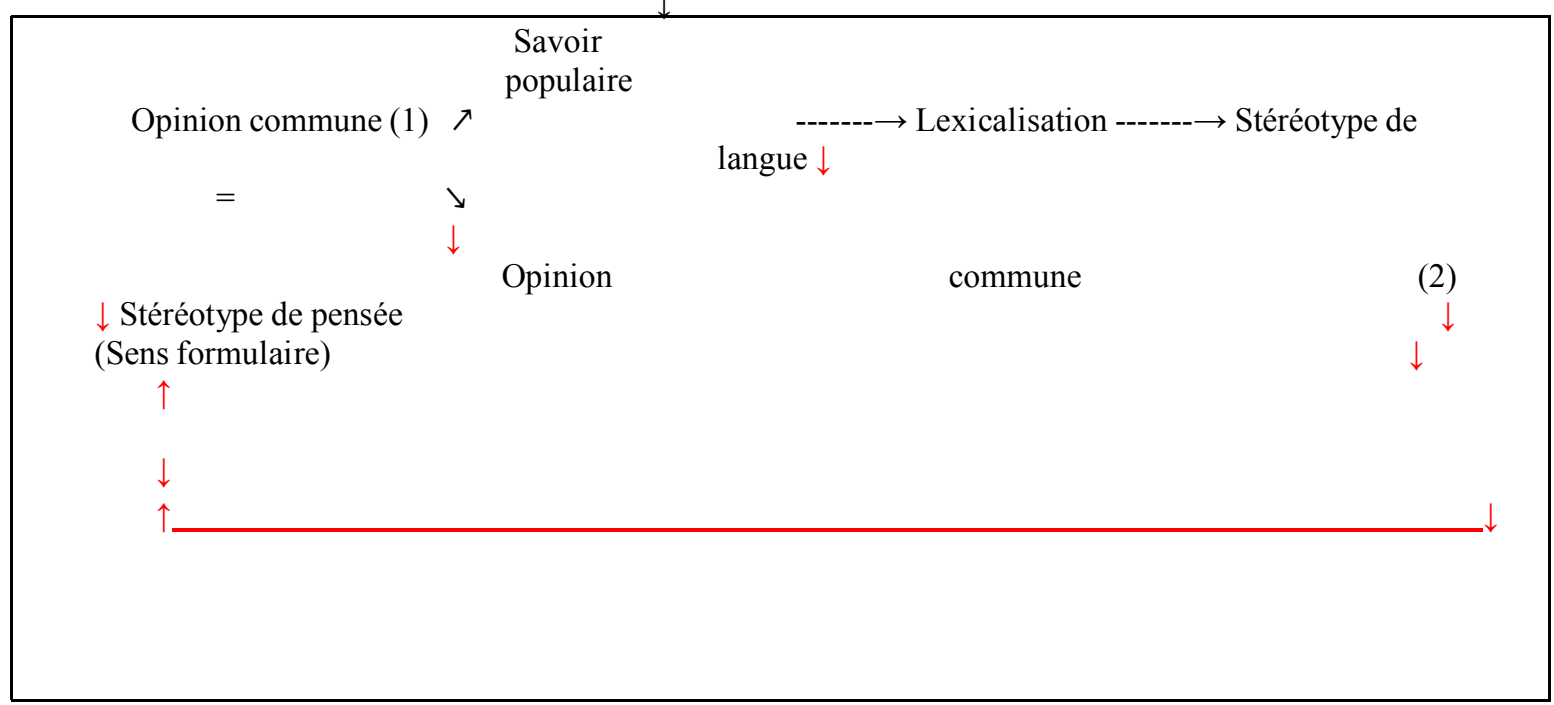

Les préceptes doxaux se sont sans doute exprimés d'abord individuellement, au moyen d'exemples plus ou moins évocateurs, puis l'usage a sélectionné et fixé par le réemploi ceux qui ont capté le mieux l'imagination des locuteurs. La preuve en est la concurrence, en diachronie, de nombre de proverbes ayant le même sens formulaire et la coexistence, en synchronie, de plusieurs formes pour un même proverbe, ou des proverbes quasi-synonymiques 16 . La réussite de l'exemplum choisi par la langue garantit en même temps la survie du stéréotype de pensée initial. Il se fixe tel quel dans l'usage et devient ainsi, aussi, un stéréotype de langue. Ce processus une fois achevé, l'idée doxale initiale transmise par le sens formulaire est restituée à la doxa, renforcée et doublement motivée du fait de son intégration au lexique.

Cette manière de concevoir l'encodage du sens formulaire dans la langue conduit nécessairement à une vision différente du rapport tripartite entre le stéréotype de pensée, la parémie en tant que stéréotype linguistique et la doxa. Ce rapport consiste en un mouvement circulaire : opinion commune (sans forme linguistique fixe) $\rightarrow$ exemplum $\rightarrow$ stéréotype de langue $\rightarrow$ stéréotype de pensée.

Cette optique montre aussi qu'indépendamment de la source doxale de son sens littéral (savoir partagé ou opinion commune), au moment de sa restitution à la doxa, le sens formulaire est toujours un stéréotype de pensée. 


\section{Clichés, lieux communs, réflexes conversationnels}

Les proverbes parlent généralement de principes humains simples et universels. Il est très rare qu'ils expriment une idée qui fustige ou critique une catégorie humaine ou sociale spécifique ${ }^{17}$

Curieusement, à peu d'exceptions près, c'est presque exclusivement dans les clichés qu'affleurent les stéréotypes ethniques et sociaux. Les clichés, tropes lexicalisés à structure syntagmatique, sont des productions orales par excellence et représentent par conséquent un phénomène fluctuant, à caractère plus dynamique que celui des proverbes. Ils constituent une classe hétérogène et composite, la moins systématique de tous les stéréotypes, et ceci pour plusieurs raisons : premièrement parce que, étant à l'origine des tropes divers qui se sont figés en langue, ils ne possèdent que rarement des traits communs se prêtant à la formalisation; ensuite, parce qu'ils présentent des degrés très variables de lexicalisation, allant des simples affinités stylistiques usuelles et par conséquent banalisées (un linguiste distingué, un grand savant, un peintre / écrivain illustre, un médecin renommé, un acteur célèbre mais plus difficilement un linguiste célèbre, un acteur, un peintre distingués, etc.), aux associations de mots stables, totalement (un illustre inconnu) ou partiellement lexicalisées (un parfait imbécile, un art consommé, un fou redoutable, etc.). On relève parmi eux des automatismes d'expression (attaquer un sujet, embrasser une cause, dompter ses instincts, vaincre son aversion, emprunter une rue) mais aussi des tropes usés et dénués de valeur parce que prévisibles : la lune aux rayons argentés, l'aurore aux doigts de rose, (les yeux sont) les fenêtres / le miroir de l'âme, des cheveux d'or/ d'ébène / de jais, des yeux de jais, les flammes de la passion, etc. Ces derniers sont des clichés stylistiques parce que, bien que fréquents, ils ne sont pas lexicalisés ; pour la plupart, leurs origines sont littéraires et non orales. Ils représentent un choix stylistique facile et irritant parce que leur répétition dénote surtout la paresse intellectuelle et un manque d'imagination et de créativité.

La lexicalisation, en effet, est un phénomène graduel (Lamiroy et Klein 2005) ; des nombreuses expressions ayant cours dans la langue parlée à un moment donné de son évolution, certaines se fixeront et bénéficieront un jour d'une mention (sinon d'une entrée) dans le dictionnaire, d'autres disparaîtront au cours du temps. Le stade de lexicalisation de l'expression, comme on le verra plus loin, constitue un critère pertinent pour l'examen de la relation de l'expression avec le stéréotype de pensée qu'elle véhicule.

Les expressions totalement intégrées à la langue, celles qui nous intéressent plus particulièrement ici, constituent un cas à part. Elles restent certes des stéréotypes mais leur emploi n'est plus ressenti comme un pis-aller expressif: bien que n'ayant plus l'effet de la surprise, elles témoignent d'une richesse de vocabulaire qui a sa valeur en discours. Elles enjolivent et revigorent le style, lui conférant un charme qui remonte dans le passé et apporte la saveur du terroir.

Que l'on pense à la poésie de Georges Brassens, que l'on pense aux innombrables expressions pittoresques d'origine orale, souvent familières : aux comparaisons par antonomase : pleurer comme une Madeleine, vieux comme Mathusalem, aux images évoquées par des locutions telles que prendre ses jambes à son cou, discuter à bâtons rompus, (parler du) coq à l'âne, se lever avec les coqs ; les expressions idiomatiques, désormais opaques: faire amende honorable, avoir voix au chapitre, reprendre du poil de la bête, battre en brèche, rompre en visière, etc.; sans parler des expressions savantes : le fil d'Ariane, la flèche du Parthe, le tonneau des Danaïdes, sacrifier un coq à Esculape, passer le Rubicon, etc. 


\section{Les stéréotypes « sociaux »}

Il est temps de considérer maintenant les stéréotypes ethniques, sociaux et racistes que l'on peut trouver dans les clichés. Ils constituent une sous-classe qui se révèle, à l'examen, plutôt restreinte par rapport à l'ensemble des clichés expressifs. C'est sans doute ce qui explique leur place marginale dans les études consacrées à la stéréotypie par les rhétoriciens, les analystes du discours, les sociologues et les sociolinguistes.

Il s'agit surtout de compléments d'adjectifs ou de noms, à effet intensif, avec la nette prédominance (cf. les exemples infra) des structures comme

$+\mathrm{N}$ et $\mathrm{N}$ de $\mathrm{N}$, très productives en français.

- ethniques : une promesse de Gascon ; radin comme un Auvergnat/ comme un Juif ; boire/ saoul comme un Polonais ; fort comme un Turc ; lent comme un Suisse ; manger en Suisse, une tête de Turc ; une querelle d'Allemands ; vivre/ parler/ errer comme des Romanichels/ comme des Tziganes; filer à l'anglaise ; le téléphone arabe ; la roulette russe ; le casse-tête chinois ; la torture chinoise, etc. ;

- occupationnels : des comptes d'apothicaires ; un travail de bénédictin; heureux comme un pape / un roi ; s'entendre comme larrons en foire ; mentir comme des arracheurs de dents; se disputer/ se battre comme des chiffonniers ; jurer comme un / des charretier(s), ragots de concierge, comme dirait mon/ ma concierge ;

- religieux: s'en foutre comme de l'an quarante (peut-être du Coran ${ }^{18}$ ); comme un mécréant ; nuit de sabbat; ronde de sabbat, faire / danser le sabbat, aller au sabbat, un sabbat de + nom $^{19}$; synagogue de $\operatorname{Satan}^{20}$, etc.

Un grand nombre de stéréotypes ont des origines historiques. C'est le cas des expressions contenant le mot nègre, datant en français de l'époque coloniale (cités dans le TLFi, entrée «nègre ») : 
Un combat de nègres dans un tunnel ; Vulg. (Il fait) noir comme dans le cul d'un nègre ; (faire) bûcher, piocher, travailler (qqn) comme un nègre ; traiter qqn comme un nègre ; (parler) petit nègre.

Proverbe. pop. À vouloir blanchir la tête d'un nègre, on perd sa lessive.

Dans les Balkans, de nombreuses expressions lexicalisées remontant à l'occupation ottomane de la région contiennent des allusions aux Turcs. Les minorités font aussi l'objet de stéréotypes linguistiques : toujours dans les Balkans, de nombreux clichés prennent pour cible les Tziganes.

Les stéréotypes concernant le genre - donc les femmes - très nombreux dans d'autres langues, comme on peut le constater en lisant les recueils de proverbes et expressions - sont eux aussi, à l'examen, relativement rares en français. A part des proverbes tels que :

Qui femme

a noise a.

Ce que femme veut, Dieu

le veut.

De femme, pâté, fruit et jambon, qui en prend peu le trouve bon. (Livre des proverbes communs du XIII ${ }^{\mathrm{e}}$ siècle $^{21}$ )

Femme qui gagne, poule qui pond, sont le diable en la maison. (Ibid.)

on trouve le lieu commun

tautologique

Une femme est

une femme

et aussi, peut-être, des contes / histoires / papotages / ragots de bonnes femmes ${ }^{22}$

\section{$9 \quad$ Y a-t-il adhésion au stéréotype de langue ?}

Pour en revenir à notre point de départ : est-il vrai que les expressions figées stéréotypées fixent les stéréotypes de pensée dans l'esprit des usagers de la langue, renforçant ainsi leur pérennité dans la doxa ? On ne peut répondre à cette question que si l'on tente d'élucider, au préalable, la question primordiale de l'adhésion de l'usager de la langue aux divers types de stéréotypes de langue. Le problème est plus vite résolu dans le cas du stéréotype de pensée : la simple prononciation en discours du stéréotype de pensée, si elle n'est pas ironique ou si l'on ne s'en dissocie pas explicitement, confirme l'adhésion du locuteur; mais il en va tout autrement du stéréotype de langue : implique-t-il l'adhésion au stéréotype de pensée correspondant? En d'autres termes, les stéréotypes linguistiques, phrastiques ou syntagmatiques, sont-ils susceptibles de perpétuer, par le réemploi, les stéréotypes de pensée qui les ont engendrés, et de contribuer ainsi à leur fossilisation dans la doxa ? Le problème se posant différemment pour les proverbes - porteurs de messages achevés - et pour les clichés syntagmatiques où le stéréotype de pensée fonctionne comme présupposé, les deux catégories seront donc traitées ici séparément 


\subsection{L'adhésion au message proverbial}

Deux arguments sont habituellement invoqués afin d'expliquer la pérennité du proverbe dans l'usage : sa supposée vérité et l'adhésion toujours renouvelée à l'enseignement qu'il contient. Toutes le formes gnomiques et le proverbe en particulier, sont censées exprimer des vérités générales ; c'est là une des sources majeures - la plus importante, peut-être - de l'autorité dont elles jouissent ; mais, comme il a été remarqué plus d'une fois, le proverbe fonde sa vérité supposée sur sa fréquence dans l'usage et non sur des preuves. "Les proverbes ont uniquement le statut de vérité par défaut » affirme Kleiber (1994: 218). La vérité du proverbe a été souvent mise en question et je ne m’y attarderai pas ici. J'estime en revanche que la question de l'adhésion est centrale pour le sujet qui nous occupe ; car, le plus souvent, la simple citation du proverbe en discours est considérée comme une preuve de l'adhésion du locuteur au stéréotype qui y est exprimé. Tamba (2000:112-114) s'interroge sur ce point et en souligne l'importance.

Elle cite F. Desbordes ${ }^{23}$ qui «considère que 'le genre sentencieux peut s'accommoder d'une platitude touchant à la viduité' car il est au service de 'rapports de force qui se soucient moins de l'expression du vrai que de l'adhésion à ce qui se donne pour vrai'. » Cependant, Meschonnic, appelé aussi à témoigner par Tamba (ibid. : 112-113), laisse entendre que ces idées qui se propagent d'un auteur à l'autre devraient peut-être faire l'objet d'un réexamen, et plus attentif. A l'idée que « le proverbe est, en toute langue, une phrase pleine de sagesse et de poids qui invite à l'adhésion», il répond : «c'est l'adhésion qui est à théoriser $»^{24}$ Or, alors que la valeur de vérité universelle de la formule est souvent contestée, l'adhésion au message proverbial, elle, semble une idée unanimement admise. La lecture des divers travaux sur la parémie révèle en effet que l'adhésion automatique au message proverbial, tout comme sa vérité et sa sagesse constituent elles-mêmes des stéréotypes de pensée.

\subsection{L’adhésion au cliché « social »}

$\mathrm{Si}$, dans le contexte de la parémie, l'adhésion constitue une question plutôt théorique, elle représente, au contraire, un problème concret et lourd de conséquences dans le cas des clichés appelés " sociaux ». Cependant, à l'examen, on constate rapidement que les différents types de clichés et lieux communs "sociaux » ne posent pas le problème de la même manière ni avec la même urgence. Il est possible, en effet, d'y distinguer deux classes de formules :

a) les stéréotypes ancrés dans une réalité périmée; il s'agit des formules faisant référence aux métiers disparus (arracheurs de dents, charretiers, chiffonniers, apothicaires), aux prétendues caractéristiques régionales dans une France désormais unifiée (Gascons, Auvergnats), ainsi que les expressions devenues opaques (travail de bénédictin, querelles d'Allemands). Ces expressions, parfaitement lexicalisées, convoquent aujourd'hui le seul sens formulaire: elles n'entretiennent plus avec les stéréotypes initiaux qu'une relation étymologique qui, si elle est motivée, en rehausse d'ailleurs l'expressivité. Paradoxalement, ces clichés d'origine orale et, pour la plupart anciennement vulgaires, anoblis par la patine du temps, ont changé de registre et ont à présent la saveur d'un français presque châtié.

b) les clichés ethniques et raciaux ${ }^{25}$; ce sont eux qui font problème. Il s'agit surtout, parmi eux, des formules les plus solidement lexicalisées et qui, par conséquent, sont déjà intégrées à la Langue. La question qu'elles soulèvent est la suivante: est-ce que le locuteur qui a en disponibilité dans son vocabulaire des expressions telles saoul comme un Polonais, fort comme un Turc, travailler comme un nègre, parler petit nègre, filer à l'anglaise, adhère par ailleurs aux stéréotypes de pensée qui les ont engendrées: les Polonais sont des ivrognes, les Turcs sont herculéens, les Noirs travaillent comme des esclaves, les Noirs parlent mal le français, les Anglais sont lâches, etc. Est-ce qu'il exprimerait en discours libre ces stéréotypes, est-ce qu'il dirait « un Nègre » en parlant d'un homme de couleur? Il serait intéressant de faire des sondages à 
ce propos mais de nombreux indices font présumer qu'il n'existe plus, pour ce type de formules, de stéréotypes équivalents dans la doxa. D'ailleurs, les listes d'expressions orales en voie de lexicalisation recueillies par Lamiroy et Klein (2005) dans l'espace de plusieurs pays francophones font état d'un nombre très restreint de stéréotypes ethniques. Il incombe aux sociolinguistes et aux sociologues de chercher les causes de cette évolution de la doxa. Dans le cadre de cette étude, l'intérêt se porte surtout sur le destin des expressions survivant aux stéréotypes de pensée qui les fondent.

Quand elle n'est plus motivée, dans certains cas, la locution subit une déformation phonétique qui la rend méconnaissable, effaçant ainsi le lien avec la figure initiale (pitinègue pour petit nègre) ; ou bien, elle est à la fois estropiée et réinterprétée sémantiquement (l'an quarante pour le $\mathrm{Coran}^{26}$ ), allant parfois jusqu'à l'absurde (parler le français comme une vache espagnole au lieu d'« un Basque espagnol »). Cependant, même quand l'expression reste parfaitement compréhensible, le locuteur est généralement conscient de sa caducité. A ce propos, je trouve particulièrement éclairante une remarque faite au cours d'une discussion, sur Internet, à propos, précisément, des clichés ethniques sur le modèle comme $+\mathrm{N}^{27}$. Elle provient d'un internaute du Maghreb, peut-être Berbère, qui dit, en parlant des expressions " radin comme un ...» : " Chez nous on dit 'radin comme un Mozabite' mais sincèrement moi je ne crois pas. »

Nombre de linguistes ${ }^{28}$ militent énergiquement pour la non inclusion des termes ethniques péjoratifs ou injurieux dans les dictionnaires ; mais peut-on, a-t-on vraiment le droit d'ignorer, dans un dictionnaire, une expression vivante dans la langue?

\section{Conclusion}

L'hypothèse que j'ai soutenue ici est qu'une expression totalement lexicalisée n'exige plus l'adhésion au stéréotype qu'elle véhicule. L'adhésion du locuteur n'est tout simplement pas pertinente dans le contexte de la langue : le lexique n'est qu'un ensemble d'outils parmi lesquels l'usager choisit, au moment de la locution, ceux qu'il juge propices à l'expression de son message.

Une formule lexicalisée est un item lexical à part entière, indépendamment du stéréotype qui l'a créée. Pour elle, comme d'ailleurs pour tout autre élément lexical, l'emploi en discours est fonction de plusieurs facteurs déterminants : le sentiment linguistique individuel du locuteur, sa sensibilité sociale, son idéologie; et surtout, son insertion en discours doit se faire consciemment, selon les circonstances de la locution, en fonction de l'interlocuteur ou du public.

Parler de l'adhésion du locuteur dans le contexte du stéréotype de langue - porteur ou non d'un message achevé - constitue donc une confusion entre la perception du rapport du locuteur à la langue, d'une part et, d'autre part, de la liberté de choix du vocabulaire en discours ; car, si le lexique est une donnée, son emploi en discours est toujours le résultat d'un choix : un choix du contenu, certes, mais aussi, et surtout, une sélection des moyens linguistiques pour l'exprimer. Et il implique toujours la responsabilité du sujet parlant. Le style, la rhétorique, l'argumentation, font appel à des opérations plus complexes. Le choix stylistique est une inspiration; le choix rhétorique est un calcul; le choix argumentatif est un exercice de logique; le choix des stéréotypes, comme d'ailleurs l'emploi de tout notre vocabulaire, est toujours une responsabilité. 


\section{Références bibliographiques}

Adam, J-M. ( 1999). Linguistique textuelle. Des genres de discours aux textes, Paris : Nathan

Amossy, R. et Herschberg Pierrot A . (1997). Stéréotypes et clichés, Paris, Nathan, coll. «128».

Amossy, R (2002) «Introduction to the Study of Doxa », Poetics Today, 23/3, pp. 369-394.

Anscombre, J-C (2000). «Parole proverbiale et structures métriques » Langages 139, pp. 6-26.

Berting, J (2001). «Identités collectives et images de l'autre : les pièges de la pensée collectiviste », Hermes, 30, pp . 41-57.

http://documents.irevues.inist.fr/bitstream/handle/2042/14516/HERMES_2001_30_41.pdf?seque nce=1

Charaudeau, P. (1995). «Le dialogue dans le modèle de discours », Cahiers de Linguistique Française, 17, Genève

: Editions de l'Université de Genève, pp. 141-178.

Charaudeau, P. et Maingueneau D. (2002). Dictionnaire d'Analyse du Discours, Paris : Seuil.

Dubroca Galin, D (2005). «Stereotypes, clichés et tournures recurrentes: des ressources linguistiques au service de la mercatique», en ROMANA GARCÍA, María Luisa [ed.] II AIETI. Actas del II Congreso Internacional de la Asociación Ibérica de Estudios de Traducción e Interpretación. Madrid, 9-11 de febrero de 2005. Madrid: AIETI, pp. 739-749. ISBN 84-8468-151-3. Version éléctronique disponible sur le site de la AIETI:

$<$ http://www.aieti.eu/pubs/actas/II/AIETI_2_DDG_Stereotypes.pd f $>$.

Farid, G (2011). « Les injures racistes ont-elles leur place dans les dictionnaires? », Chroniques de langue, $8 / 1$, p. 26

http://www.btb.termiumplus.gc.ca/tpv2guides/guides/chroniq/index-

fra.html?lang=fra\&lettr=indx autr8YsI9VT8NQ40\&page=9kLtCh0HKRQw.html

Fournier, P N (2010). «La stéréotypie, un avatar de communication incontournable dans l'enseignement apprentissage d'une langue-culture étrangère. Essai de mise au point conceptuelle. Synergies Pays riverains du Mékong, no.2, pp. 47-65, mis en ligne 25 janvier 2011

Kleiber, G. (1994) : "Sur la définition du proverbe", en Nominales. Essais de sémantique référentielle. Paris: Armand Colin, pp. 207- 225.

Kerbrat-Orrecchioni, C. (1980). L'énonciation, de la subjectivité dans le langage, Paris: Armand Colin.

Lamiroy, B. et Klein JR (2005). " Le problème central du figement est le semi- figement » http://www.ling.arts.kuleuven.be/franitalco/papers/LamiroyKlein2005.pdf

Lecolle, M. (2006). "Changement dans le lexique — changement du lexique: Lexicalisation, figement, catachrèse ", Cahiers de praxématique [En ligne], 46 |2006, mis en ligne le 01 décembre 2009, consulté le 04 août 2013. URL : http://praxematique.revues.org/595

Paveau, M-A. (2003). «L'entrée DOXA : pour un traitement rigoureux d'une notion floue », Mondialisation(s) 71, pp. 176-181.

Sarfati, G E. (2000). «De la philosophie et l'anthropologie à la pragmatique : Esquisse d'une théorie linguistique du sens commun et de la doxa », Actes de la journée d'étude du 17 mars 2000 du Groupe d'Etudes en Psycholinguistique et Didactique, Cognition, langue et culture, éléments de théorisation didactique, Paris, p. 3952, consulté en ligne formes- symboliques.org/IMG/doc/doc-144.doc

Sarfati, G E. (2002a). «Cultural Study, Doxa, Dictionaries: The Case of Jewish Identity», Poetics Today 23/3, pp. 489-512

Sarfati, G E. (2002b). "Aspects épistémologiques et conceptuels d'une théorie linguistique de la doxa », 
in Amossy R. \& Koren R. (dir.), Après Perelman : quelles politiques pour les nouvelles rhétoriques ?, Paris : l'Harmattan

Schapira, C. (1999). Les stéréotypes en français : proverbes et autres formules, Paris - Gap : Ophrys.

Schapira, C. (2012). «La Tautologie dans l'énoncé parémique» in Anscombre, J.-Cl., Darbord, B., Oddo, A. (éd.), La Parole exemplaire. Introduction à une linguistique des proverbes, Paris : Armand Colin, pp. 95-113.

Tamba, I. (2000). «Formules et dire proverbial», Langages 139, pp.110-118.

Tamba, I. (2011). «Sens figé : idiomes et proverbes », (2011), in Anscombre, J.-C. \& Mejri ,S. (éd.), Le figement linguistique : la parole entravée, textes réunis par J.-C. Anscombre et S. Mejri, H. Champion, pp. 109-126

${ }^{1}$ Cf ., parmi d'autres, Dufay (2004: 26) : le stéréotype est « un signe banal, prévisible, rebattu, usé et donc dénué de valeur »; mais Dufay, comme d'ailleurs bien d'autres auteurs (à la suite d'Amossy et Herschberg - Pierrot 1997), considère comme des stéréotypes de langue les seules collocations non lexicalisées résultant d'associations stylistiques fréquentes et devenues par conséquent quasi automatiques.

${ }^{2}$ Le TLFi - http://atilf.atilf.fr/

${ }^{3}$ Charaudeau et Maingueneau (2002), Dictionnaire d'Analyse du Discours, entrée Doxa

${ }^{4}$ Pour un aperçu compréhensif de la notion et de son développement historique voir Ruth Amossy et Meir Sternberg(éd.), Doxa and Discourse ; How Common Knowledge Works, Poetics Today 23/3, Fall 2002.

${ }_{6}^{5}$ Cf. le titre de l'article de Paveau (2003).

6 La doxa s'exprime aussi par le comportement, les actions, les décisions des individus. C'est ce qui en fait un objet d'étude privilégié des sciences sociales, de l'anthropologie, etc.

${ }^{7}$ Adam conçoit la schématisation communicative en termes d'images. Parmi les six types d'images de base proposés, figurent les images de la langue attribuées à l'interlocuteur ou les images que l'interlocuteur s'attend que l'on produise.

${ }^{8}$ Encyclopedia universalis, entrée «Stéréotypes sociaux» 2: La pensée stéréotypée. Consulté en ligne le 2 septembre $2013: \mathrm{http} / /$ www.universalis.fr/encyclopedie/stereotypes-sociaux/2-la-pensee-stereotypee/

${ }^{9}$ On cite, à ce propos, dans plusieurs ouvrages, l'exemple suivant : " Ma belle-mère vient chez moi aujourd'hui mais elle est gentille. » La construction adversative ne demande pas d'explication, parce qu'elle repose sur le stéréotype doxal selon lequel les belles-filles n'aiment pas leurs belles-mères.

${ }^{10} \mathrm{Ceci}$ exclut de la classe les collocations provenant d'affinités stylistiques dues à un usage fréquent mais non lexicalisées.

${ }^{11}$ Tamba (2011 :118) remarque en effet que le stéréotype « apparaît [...] comme une façon de parler marquée par rapport à son équivalent standard». «Sans doute n'est-ce pas un hasard si de tels idiomes concurrencent le vocabulaire neutre », ajoute-t-elle. Tamba parle des expressions idiomatiques en général mais ses exemples sont des stéréotypes de langue : casser sa pipe et passer l'arme à gauche pour mourir.

12 «Au-delà de la question des seules unités lexicales, cette dialectique entre usure et créativité, [...] caractérise aussi bien le destin des figures mortes que le figement [...] écrit Lecolle (2006 : §32).

${ }^{13}$ Cf. la typologie proposée dans mon ouvrage Les Stéréotypes en français (Schapira 1999).

${ }^{14}$ Ces proverbes, assez abondants en français, représentent une catégorie négligée par les études parémiques omission d'autant plus étonnante qu'ils forment un corpus dont les caractéristiques sont susceptibles d'être formalisées. Cf. Schapira (2012).

15 Entrée LIT, consulté en ligne : http://www.littre.org/definition/lit

${ }^{16}$ Cf., par exemple,

Rien ne sert de courir il faut partir à point. / Qui trop se hâte reste en chemin. Tel père tel fils. / Petit poussin chante comme le coq lui apprend.

${ }^{17}$ Les stéréotypes concernant le supposé mauvais caractère des femmes constituent une exception mais ils sont relativement rares en français :

Qui femme a noise a.

Ce que femme veut, Dieu le veut. 
A en croire les recueils des proverbes du monde, ce genre de commentaire sur les femmes est bien plus fréquent dans les sociétés orientales.

${ }^{18}$ L'origine de cette expression, restée mystérieuse, a donné lieu à de nombreuses hypothèses. L'une d'entre elles (Maurice Rat, Dictionnaire des locutions et expressions traditionnelles) attribue " l'an quarante " à une déformation de " al-Koran ». Rey et Chantreau (Dictionnaire des expressions et locutions) rejettent cette possibilité par manque de preuves mais en réalité aucune des autres solutions que l'on a pu proposer n'offre de preuves solides en sa faveur. (cf. http://www.langue-fr.net/spip.php?article59). Certains linguistes optent pour un croisement entre plusieurs objets visés, dont le Coran.

${ }^{19} \mathrm{Cf}$. TLFi entrée sabbat : B1 [À cause de l'interprétation malveillante du sabbat juif faite par les chrétiens] (Occult.) Assemblée nocturne de sorciers et de sorcières, tenue dans un lieu désert souvent élevé, dans laquelle le culte rendu au diable, les danses et les orgies rappellent ceux de l'antiquité païenne; pratiques auxquelles on s'y livre..

${ }^{20}$ TLFi entrée synagogue : A1 b) $\beta$ [Dans le monde chrét.] Synagogue de Satan. [P. allus. à Apoc. II, 9] Ceux qui sont dans l'ennui, les hérétiques; ceux qui se conforment à l'ancienne loi, par opposition à la loi nouvelle (d'apr. GUERIN Suppl. 1895) ; - En partic. [Chez certains opposants cath.] La franc-maçonnerie.

${ }^{21} \mathrm{http}: / /$ www.mon-poeme.fr/proverbes-francai13-siecle/\#

${ }^{22}$ Cf. cette citation de Jules Romains illustrant dans le TLF (entrée femme (3.C. Rem.d)) la différence entre femme et bonne femme :

[...] dans la différence entre les « femmes » et les « bonnes femmes », la question d'âge n'intervenait presque pas. Telle personne de cinquante ans, pourvu qu'elle fût coquette, bien mise, qu'elle eût une certaine peau, un certain regard, un certain parfum, lui apparaissait sans hésitation comme une «femme »; et telle concierge de vingt-cinq ans de la rue de la Goutte-d'Or, qui balayait son vestibule, dépeignée, dépoitraillée, la robe poussiéreuse, l'œil habité par des pensées de ménage ou de hargne conjugale, était promue d'emblée au rang de "bonne femme ". ROMAINS, Hommes bonne vol.,1932, p. 268.

${ }^{23}$ Desbordes, F., 1980, «Les vertus de l'énoncé », La licorne, pp. 65-84, cité par Tamba (2000 $: 113)$.

${ }^{24}$ Meschonnic, H., 1976, «Les proverbes actes de discours », Revue des Sciences Humaines, XLI, 163, p. 419. Italiques dans le texte.

${ }^{25}$ J'élimine de la discussion les insultes directes ou indirectes (comme un Arabe, comme un Juif, comme des Tziganes, comme un Suisse, comme un Belge, etc.) qui, d'ailleurs, constituent des collocations ne présentant qu'un faible degré de cohésion.

${ }^{26}$ A supposer que cette interprétation de l'origine de l'expression soit correcte.

${ }^{27}$ http://answers.yahoo.com/question/index?qid=20080303025205AAnZ6 D

On y trouve, bien sûr, saoul (bourre) comme un Polonais, fort comme un Turc mais aussi têtu comme un Irlandais, voleur comme un Italien, radin comme un Israélien / comme un Hollandais, lent comme un Suisse.

${ }^{28}$ Farid (2011), parmi d'autres, avec l'espoir que « ces injures racistes tomberont en désuétude si les lexicographes ne perpétuent pas leur consignation servile »; mais son article traite des mots argotiques injurieux (boche, amerloque, cinetoque, polaque, youpin, bicot, etc.) et non des stéréotypes lexicalisés, dont l'exclusion du dictionnaire serait impensable. 